Revista Calidad en la Educación Superior

Programa de Autoevaluación Académica

Universidad Estatal a Distancia, Costa Rica

ISSN 1659-4703

revistacalidad@uned.ac.cr

\title{
DIAGNÓSTICO SITUACIONAL DE LA FORMACIÓN DE DOCENTES EN HONDURAS
}

\author{
SITUATIONAL DIAGNOSIS OF TEACHER TRAINING IN HONDURAS
}

Luis Diego Chacón Víquez' Idchacon@ujn.edu.hn Universidad Jesús de Nazareth, San Pedro Sula, Honduras

\author{
Volumen 7, Número 1 \\ Mayo 2016 \\ pp. $205-232$
}

Recibido: 30 de agosto, 2015

Aprobado: 10 de abril, 2016

\footnotetext{
${ }^{1}$ Luis Diego Chacón Víquez. Docente de la Universidad Jesús de Nazareth, San Pedro Sula, Honduras. Candidato a Doctor en Educación de la Universidad de Costa Rica. Correo electrónico: Idchacon@ujn.edu.hn
} 


\section{Resumen}

Este diagnóstico situacional, enmarcado dentro de la metodología de la revisión bibliográfica, nace como parte del programa Latinoamericano de Doctorado en Educación de la Universidad de Costa Rica. En este documento se analiza dentro de un contexto sociocultural e histórico el proceso de desarrollo que ha vivido la formación docente en la región Latinoamérica, desde el inicio de los procesos independentistas en el siglo XIX, por medio de las escuelas normales, hasta la profesionalización de los maestros en el plano del nivel terciario a través de universidades e institutos pedagógicos, a mediados del siglo $X X$ y que continúan en nuestros días. Mucha de la preocupación que se presenta en el análisis de los diferentes contextos se relaciona con la mejora de las condiciones en las que se da la formación docente. Se han implementado cambios buscando que el desempeño docente siempre esté en sintonía con los desarrollos globales característicos de las sociedades en constante auge de crecimiento. Estos cambios en el contexto sociocultural e histórico han demandado los continuos replanteamientos que influyen notoriamente en el análisis de los procesos de formación de docentes. Si bien se utiliza el marco de referencia latinoamericano, este estudio se centra en la situación que ha vivido y vive hoy en día Honduras en este tema, al tiempo que analiza los efectos socioculturales e históricos que han generado los cambios sufridos en los procesos de formación de docentes. Para la realización de esta investigación se plantea la pregunta de investigación: ¿Cómo ha sido el proceso de la formación de docentes en Honduras desde las escuelas normales hasta el nivel terciario de la educación? La estructura del documento sigue las etapas de realización e incluye una Introducción, un análisis histórico de los procesos de formación docente en América Latina, un desarrollo y las conclusiones respectivas.

Palabras clave: formación Inicial, Formación docente, escuelas normales, reformas educativas, profesionalización docente, América Latina.

\section{Abstract}

This situational analysis, framed within the methodology of the literature review, is part of the curriculum of the Latin American Doctoral Program in Education at the University of Costa Rica. In this paper, we analyze within a sociocultural and historical context, the process of developing teacher training has lived in the Latin American region, since the beginning of the processes of independence in the nineteenth century, through the normal schools, to professionalization of teachers at the tertiary level through college and university teaching in the mid-twentieth century and continue today. Much of the concern that occurs in the analysis of different contexts relate to the improvement of conditions in which teaching is given formation. 
Seeking changes have been implemented that teacher performance is always in tune with global developments characteristic of societies in constant growth boom. These changes in the sociocultural and historical restatements have demanded that influence continued well into the analysis of the processes of teacher training While using the Latin American framework, this study focuses on the situation was and is today Honduras days on this issue, while analyzing cultural and historical effects that have generated the changes undergone in the process of teacher training. To carry out this research raises the research question: How was the process of teacher training in Honduras from normal schools to tertiary level of education? The document's structure follows the stages of implementation and includes an introduction, a historical biography of the processes of teacher training in Latin America, middle, and the respective conclusions

Keywords: Initial training, Teacher training, pedagogic school, education reform, teacher professionalism, Latin America.

\section{Resumo}

Esta avaliação da situação, enquadrado dentro da metodologia da revisão da literatura, foi criado como parte do Doutorado Latino-americano em programa de Educação da Universidade de Costa Rica. Este documento é analisado dentro de um contexto sócio-cultural e histórica do processo de desenvolvimento que viveu a formação de professores na região da América Latina desde o início dos movimentos de independência no século XIX, através de escolas normais para profissionalizar professores ao nível do ensino superior através de universidades e instituições de ensino em meados do século XX e continua até hoje. Grande parte da preocupação apresentada na análise dos diferentes contextos está relacionada com a melhoria das condições em que tem lugar a formação de professores. As alterações foram implementadas buscando que o desempenho do professor está sempre em sintonia com a evolução global característicos das sociedades em constante boom de crescimento. Essas mudanças no contexto sócio-cultural e histórica processaram o notoriamente continuamente sendo que a influência da análise dos processos de formação de professores Enquanto quadro de referência latino-americana é utilizado, este estudo centra-se sobre a situação que viveu e vive agora Honduras dia sobre esta questão, ao analisar os efeitos sócio-culturais e históricas que geraram as mudanças sofridas no processo de formação de professores. Para realizar esta pesquisa levanta a questão de pesquisa: Como foi o processo de formação de professores em Honduras a partir de escolas normais para o nível superior de educação? A estrutura do documento segue as fases de implementação e inclui uma introdução, uma análise histórica dos processos de formação de professores na América Latina, o desenvolvimento e respectivas conclusões. 
Palavras-chave: formação inicial, formação de professores, faculdades de formação de professores, reformas educacionais, profissionalização docente, América Latina.

\section{Justificación}

Conforme se han venido forjando los diferentes cambios en general, se ha notado un aumento en las acciones realizadas en América Latina para capacitar a las personas para la ejecución de la labor de educar a las siguientes generaciones, a partir de la creación de las escuelas normales, a mediados del siglo XIX. Dichas escuelas de formación de docentes nacieron con la intención de formar ciudadanos destacados -personas que se necesitaban para impulsar los cambios de los países debido a los procesos de independencia que se dieron años antes en muchos países del continente- más allá de transmitir las habilidades básicas de la lengua, las matemáticas o las ciencias sociales.

Consonante en paso de los años, las mismas demandas sociales, concernientes al conocimiento y de necesidad de profesionalización, han generado las reformas de los sistemas educativos de los países, y los temas relacionados a los procesos de formación de docentes no ha sido la excepción, ya que se tomaron medidas para evitar el rezago de los países de América Latina. Debido a este aplazamiento observado, se fueron creando los centros universitarios especializados en formación de docentes como respuesta a los cambios históricos y sociales, mismos que se desarrollarán en este trabajo.

En este diagnóstico situacional se pretende utilizar la revisión bibliográfica de los diferentes contextos y latitudes, especialmente, las que hacen referencia a Latinoamérica y Centroamérica, a manera de abordaje metodológico. Este documento analiza las transformaciones que han vivido los centros de formación 
docente en América Latina desde mediados y finales del siglo XIX hasta nuestros días, que según Aranda (2006) "una revisión bibliográfica escrita no consiste en una serie de citas o extractos" (p. 2). Busca de alguna manera organizar, estructurar y sintetizar las referencias, con lo que se busca generar una compilación que permita al investigador conocer el estado actual del tema que está desarrollando.

Se da un recorrido por la historia y las experiencias vividas con las escuelas normales hasta los procesos constitutivos de las universidades e institutos pedagógicos. Finalmente, la estructura del documento incluye una introducción, un análisis histórico de los procesos de formación docente en América Latina, un desarrollo que toma en cuenta la historia de la formación docente en el continente, desde las escuelas normales hasta las universidades y de qué manera esto ha influido en los contextos socio culturales; y las conclusiones respectivas.

\section{Los inicios de la formación docente}

A través de la historia, los primeros modelos implementados en países como Alemania y Francia, concernientes a la formación de docentes, se relacionaron con los intentos de los gobiernos de transmitir a través de los nuevos maestros las normas generales para la difusión de los principios y teorías pedagógicas en las escuelas primarias donde eran asignados los recién egresados de las escuelas normales.

Para iniciar este recuento es necesario hacer un recorrido hacia atrás en la historia hasta posicionarse en Alemania, ya que, es en ese país donde se remarca el comienzo de las escuelas cuya finalidad era la formación de docentes. Según lo plantea Alves (2009) los inicios de estas escuelas de formación de maestros se atribuye a "Comenius y Ratke, aunque su contribución fue más indirecta que directa" (p.19). Todo esto en la primera mitad del siglo XVII. Hace referencia Alves, citando 
a Escolano y Fernández, que la atribución real de ser el primero en formar un centro de formación de maestros se atribuye a Shiemneyer quién en 1732 fundó "la primera escuela normal con carácter oficial y Herker, así mismo en 1748 estableció una en Berlín, personaje que ejerció gran influencia en todas las demás" (p.16).

\section{Análisis socio histórico de la formación docente América Latina}

Para América Latina el proceso de formación de docentes se dio muy relacionado a los procesos de independencia que se venían forjando en prácticamente todas las naciones. En el caso de Uruguay, la educación fue fundamental para los procesos precedentes de la consolidación de la Nación, tal y como lo mencionan Caraballo, Chichiraldi y Curto (2004) "Esa suerte de "estado docente" (...) (sic) acompañó lentamente el apogeo y también la decadencia del Estado de bienestar a lo largo del siglo XX". (p.1)

En Uruguay, para Caraballo, Chichiraldi y Curto (2004) no es hasta 1885 que se inicia el "camino de profesionalización de la carrera docente magisterial con la inauguración del nuevo local que diera albergue a los Institutos Normales de Montevideo ya existentes, Stagnero de Munar y Sánchez. (p.6). Un hito que marcó la historia en América Latina, fue que el país contara con un equipo de docentes de "educación básica primaria 100\% profesionalizado y titulado". (p.7)

En Colombia la formación de docentes inicia a partir de la apertura de las escuelas normales en 1872, liderada por una orden religiosa en uno de los barrios de Bogotá, aunque la primera escuela oficial creada por el estado fue la escuela normal de varones y cuya finalidad era, desde la óptica de Báez (2004) la "formación de profesores para las escuelas normales de los Estados Soberanos". (p.181). La escuela se fundamenta en el método Pestalozziano donde la obligación de la 
formación de los docentes recaía en la figura del director, que en este caso era, el profesor Ernesto Hostchick, de origen alemán.

En el caso de Chile, según Avalos (2003) "la primera forma institucional de formación docente en el Chile independiente surgió con el establecimiento de una Escuela Normal de Preceptores en 1842" (p.2) siguiendo el modelo de la escuela normal francesa. Al igual que otros países de la región, Chile recibió una gran influencia alemana que a la vez fungió como base para la primera institución dedicada a formar docentes para el nivel secundario, fundada en 1889.

Para el caso de Bolivia, llama la atención lo tardío en el proceso de incursión del estado en la formación docente, al mismo tiempo que, la hegemonía de la formación en las últimas décadas del siglo XIX fueron dominados por la Iglesia. No es hasta la fundación de la Escuela Nacional de Maestros en 1909, llevada a cabo por el Partido Liberal con la consigna de volver laico el sistema escolar, que se inicia este proceso, siendo la primera institución no manejada por órdenes religiosas dedicada a formar los futuros profesores; teniendo la clara intención de ejecutar una formación integrada, aunque, con la preminencia de contenidos científicos y técnicos. Para Lozada (2004) "Los futuros maestros que se formarían en ella debían dominar contenidos especializados, los cuales se trasmitiría a los estudiantes bolivianos a través de una disciplina rígida". (p.54)

Referente a Brasil en 1827, la Asamblea General Imperial decreta una ley que tiene como objetivo la ordenación de la instrucción pública y de los conocimientos necesarios a la formación docente para las escuelas primarias, pero no es hasta que en 1830 que, según Alves (2004) "empezaron a surgir proyectos de escuelas normales en diferentes provincias brasileñas. Entre las primeras están aquellas localizadas en las provincias de Minas Gerais (1835), Rio de Janeiro (1835), Bahía 
(1836) y São Paulo (1846)", (p.21), con la finalidad de ejercer control sobre las actividades pedagógicas implementadas por los maestros de las escuelas primarias, sustituyendo lo que Alves (2009) denominó el saber del oficio por una base científica.

La formación de docentes en Argentina inicia con la primera Escuela Normal que se fundó en Paraná, provincia de Entre Ríos en 1870. Dicho establecimiento fue organizado en dos cursos, tal y como lo mencionan Cámpoli, Michati y Gorboff, (2004), quienes citan a Solari:

El curso normal, destinado a dar no solamente un sistema de conocimientos apropiados a las necesidades de la educación común en la república, sino también al arte de enseñar y las aptitudes necesarias para ejercerlo y el curso de aplicación, que además de brindar instrucción elemental a niños de ambos sexos, sería utilizado por los alumnos-maestros para realizar la práctica de la enseñanza" (p.11)

Para finales del año 1899, en Ecuador se estima necesario fundar institutos de formación para maestros, para lo cual se inició con un proceso de contratación de profesores extranjeros con la finalidad, según Abendaño (2004), de "crear la Escuela Normal de Varones que se establecería en Quito y a las profesoras Alice Fischer y Rosina Kinsman para la Escuela Normal de Señoritas. En ese mismo año, 1899, dicta el Acuerdo Ministerial de creación de las Escuelas Normales". (p.10). En 1901 se expide el reglamento general de las Escuelas Normales, formadoras de maestros.

\section{Revisión histórica de la formación docente en México y Centro América}

En el caso particular de México, antes que finalizara la primera mitad del siglo XIX, la formación de maestros tenía la característica principal de estar 
descontextualizada del ámbito socio político que la época determinaba, esto en disonancia con lo que se venía observando en la mayoría de la región, relacionado a los a procesos de impulso de los países post-independencia. No es sino hasta que se logra la Independencia, en 1823, que el gobierno, como ente ejecutor de las políticas públicas, reconoce, según Santillán (2003), "la necesidad de crear establecimientos de instrucción con ideologías que estuvieran en consonancia con el sistema político (...) creando lo que para la época fuera la única opción para la formación de maestros: la Escuela Nacional Lancasteriana. Para 1900 funcionaban 45 escuelas normales en el país". (p.1)

Ya en Centroamérica los diferentes países hacían sus esfuerzos de profesionalizar a sus maestros, en el caso de Costa Rica, en el año 1886 se da un gran paso en el camino a la profesionalización docente cuando se crea la primera escuela normal, denominada Central, con la convicción de formar docentes varones. Para Salazar (2003), pretendía "formar maestros imbuidos de un sentido útil de las materias, aplicadas especialmente a las actividades comerciales e industriales" (p.210); pero dicha iniciativa tuvo una corta duración de dieciocho meses únicamente. Al igual que se dio en otras latitudes del continente, las corrientes impulsoras de estas reformas estaban relacionadas, según Vargas (2008), al "positivismo y liberalismo de la época". (p.82)

En el caso de Nicaragua, el país sufrió una serie de situaciones políticas, sociales y económicas durante los inicios del siglo XIX que afectó considerablemente el desarrollo de la educación y de los procesos encargados de formar docentes. Los puestos para las personas que iban a encargarse de formar a las nuevas generaciones, que para Lucio (2008) "tuvo un carácter eminentemente selectivo desde el punto de vista social" (p.32). Llama la atención que lo tardío que el Estado tomó las riendas de la formación de los docentes en Nicaragua, pues no fue hasta 
1948 que se logra implementar un sistema de formación para maestros a través de las escuelas normales, con la Ley de Fundación de las Escuelas Normales.

En Guatemala la educación estuvo monopolizada, al igual en otros países de la región, por la iglesia católica. Debido a los cambios relacionados con los procesos de independencia que se venían dando en toda Centroamérica, en Guatemala se abordaron las carencias en el tema de formación de docentes con el fin de que los maestros fueran capaces de formar las futuras generaciones educadas y con virtudes; pero desde el punto de vista laica, gratuita y obligatoria. No es hasta que se funda la Escuela Normal de Primeras Letras en 1837 que se inicia este proceso, según Preal/Flacso (2008) "la formación de educadores en servicio con énfasis en el Método Lancasteriano". (p.32).

En Panamá los inicios relacionados con la formación de docentes se dan con la apertura, en 1872, de la escuela normal para varones. Al igual que en otros países de América Latina, la influencia que se recibe es de pedagogos europeos para que administren los procesos de educación a los nuevos maestros. No es sino que hasta en 1873 que el estado toma las riendas de la formación de docentes con la apertura de la Dirección de Instrucción Pública. Báez Osorio (2004) mencionan que "Para 1880 el estado había ampliado la cobertura educativa y aumenta la creación de escuelas normales para cubrir la demanda de maestros". (p.200)

Para el caso de Honduras, según datos históricos y desde la óptica de Salgado (s.f) el nacimiento o inicio de los "procesos de formación de docentes en Honduras datan de 1875 en una ciudad de Gracias Lempira" (p.1). Durante estos años, y hasta mediados del siglo XX, la única opción de brindar instrucción formativa para maestros fue a través de las escuelas normales. Algunas décadas después, ya en el siglo XX, específicamente entre 1905 y 1906, se fundan en Tegucigalpa, capital 
de Honduras, según Salgado "En las aulas de estas Escuelas Normales se formaron los maestros que dirigieron la educación en Honduras a principios del siglo $X X " .(p .1)$.

\section{Formación Docente en el nivel terciario}

Conforme los adelantos científicos, sociales y laborales fueron incursionando en la vida de las personas durante el siglo XX; igualmente, la formación de docentes tuvo que acoplarse a los cambios y requerimientos que se iban dando conforme el siglo seguía transcurriendo. La formación inicial de docentes pasó paulatinamente de la tradicional escuela normal a los diferentes centros de nivel superior que fueron abiertos como: Institutos Superiores, Facultades de Educación de las Universidades y las Universidades Pedagógicas; buscando con esto consolidar los procesos de formación de docentes en América Latina.

Es así como los cambios se han ido incorporando en las diferentes agendas sociales, políticas y económicas de los diferentes países de la región, generando una serie de situaciones que han marcado los procesos relacionados con la formación de formadores, y con esto, forjando innumerables mejoras en los métodos de enseñanza, se plasman los diferentes cambios vividos por los países de América Latina.

Así bien, en Argentina la formación docente inicial está centrada en centros de formación de formadores como ser institutos superiores para-universitarios, así como instituciones del nivel terciario que se desempeñan de una manera paralela.

Por otra parte, se dan una serie de reformas en los países de Latinoamérica que genera cambios importantes en las estructuras educativas, tal y como lo expresa Pérez (1998) quién dice que: 
(...) la preocupación por el desarrollo profesional del docente, tanto en sus aspectos de formación como de ejercicio de su práctica, condiciones de trabajo, consideración social, (...) se ha convertido no sólo en un problema político, administrativo y técnico, sino en un importante objeto de investigación, debate público y desarrollo legislativo. (p.179)

Por lo que cabe preguntarse ¿Qué tanto influyen los docentes en las sociedades? ¿Cuál es el verdadero papel de los maestros?, siguiendo las palabras de Pérez (1998), y tras haber analizado un sinnúmero de publicaciones relacionadas con el tema, es fácil visualizar que el papel o bien el rol que juegan los docentes en la formación de nuevas generaciones resulta fundamental en el desarrollo de las naciones, pues es a partir del trabajo ejecutado por miles de docentes que se transmiten conocimientos, valores, ideologías, metodologías e incluso visiones de país.

Más allá de visualizar la docencia como un trabajo de transmisión de conocimiento, la importancia de la profesionalización, según Pérez Gómez radica en que "el desarrollo profesional de los docentes en la actualidad debe analizarse en la complejidad de sus manifestaciones divergentes e incluso contradictorias" (p.183). De ahí que sean grandes los esfuerzos que se realizaron y se realizan actualmente para dar las herramientas necesarias a la fuerza laboral docente de los países.

Tomando en cuenta la serie de cambios y reformas dadas en los diferentes países del continente, se ha visto como paulatinamente se fue fortaleciendo, con forme avanzaba el siglo XX, la cobertura de instituciones encargadas de formar docentes. Afirma Pérez Gómez que "los procesos de formación y perfeccionamiento han 
adquirido el status universitario, y en la mayoría de los países desarrollados hasta nivel de licenciatura" (p.181)

En Uruguay por ejemplo, la formación de maestros, aún en estos días, se da en los institutos normales de Montevideo y en los institutos de formación docente que se localizan en todas las regiones del país. Tal y como hacen referencia Caraballo, Chichiraldi y Curto (2004) "Para la obtención del título Magisterial, existen dos caminos: graduarse en los Institutos Normales de Montevideo, o bien en los Institutos de Formación Docente del Interior del País, opción magisterio". (p.16) En Bolivia, poco a poco se han ido consolidando los institutos normales superiores que sustituyen a las antiguas escuelas normales, así como en la Universidad Pedagógica. Según Lozada (2004) "Actualmente existe una Universidad Pedagógica dedicada exclusivamente a la formación de maestros, 5 Institutos Normales Superiores ubicados en capitales de Departamento". (p.47)

En Ecuador, a partir de 1960, las estructuras de formación docente denominadas escuelas normales, pasan a ser Institutos Pedagógicos que son instituciones de educación superior no universitarias y que según Avendaño (2004) "Actualmente existen en el país 25 Institutos Pedagógicos, IPED's [así como una] (...) Universidad Pedagógica”. (Pp.11-14)

Al igual que en otros países de la región, en Chile las escuelas normales desaparecen, cuando la dictadura de Augusto Pinochet toma las riendas del país. Tras argumentar deficiencias económicas y políticas se cierran las escuelas normales, y se trasladan los estudiantes a diferentes universidades. Ávalos (2003) menciona que el "Hecho significó la materialización del viejo anhelo de los profesores normalistas por una Escuela Única de Formación Pedagógica para 
maestros primarios y secundarios al tiempo que la destrucción del concepto de normalismo que había nacido en el siglo XIX" (p.6)

En Colombia se encuentra el fenómeno que las escuelas normales están en proceso de desaparecer, al tiempo que nacen las escuelas normales superiores asociadas a las diferentes facultades de educación de las dos Universidades pedagógicas: la Pedagógica Nacional y la Pedagógica y Tecnológica de Colombia. En este caso y según un estudio realizado por la IESALC (2006) "se asistió tanto a un decremento del número de normales como del de sus egresados, y a un aumento considerable de la matrícula en educación secundaria y en las Universidades Pedagógicas". (p.20)

Para Brasil, se puede mencionar que aún hoy en día se mantiene el modelo de las Escuelas Normales y Universidades para los primeros años de la educación básica y para los años posteriores.

En Argentina, la formación de formadores está bajo la tutela de Institutos Terciarios no-universitarios, Debido a una serie de reformas producidas después de diferentes análisis del contexto nacional e internacional se decide, a partir del año 1969, que la formación de los docentes que anteriormente se brindaba en las escuelas normales, pase bajo el control del nivel terciario; para la formación de docentes en los niveles inicial y primario. Cámpoli, et al, (2004) mencionan que,

Estos cursos se denominaron profesorados de enseñanza primaria y pre-primaria, y funcionaron anexos a las escuelas normales. Estas por su parte, comenzaron a emitir títulos de bachiller con distintas orientaciones, entre ellas la pedagógica. (p.15) 
En el caso de México, a partir de 1984, las escuelas normales pasaron a ser consideradas como instituciones del nivel superior; en 1978 por Decreto Presidencial se da la creación de la Universidad Pedagógica Nacional, y con esto ambas instituciones se encargan de la formación de docentes. Al mismo tiempo, menciona Santillán (2003) que "la Dirección General de Educación Normal (DGEN), la cual en 1984 incorporó sus escuelas al modelo de educación superior, ofreciendo estudios a nivel licenciatura para la formación de profesionales de la educación" (p.3).

La formación docente debe analizarse desde la perspectiva que todo debe evolucionar al ritmo de los cambios socioculturales del que todos los días somos testigos. Y es que es tan importante el papel de los docentes en las sociedades y el papel que ejercen dentro de su función y labor social que son tema de análisis continuo, tal y como lo menciona Rojas, et al (2001) citando a Sol

Es importante recordar que casi a diario, los medios de comunicación social publican artículos o propician programas en los que se discute ampliamente sobre el tema de la educación, todos en un esfuerzo por aportar y proponer soluciones. (p.2)

En el caso de Panamá, no se encuentra evidencia de la existencia de una universidad especializada en Pedagogía, las escuelas normales juegan un papel preponderante. Si bien es cierto, han existido esfuerzos para crear un centro del nivel terciario especializado en pedagogía, los grupos estudiantiles de la Universidad de Panamá han realizado protestas para que no se dé la aprobación. La formación a nivel superior de docentes nace con la apertura de carreras de prescolar y primaria. A partir de 1970, la Universidad de Panamá, según Santamaría (1994) "también expide el título de Licenciatura en educación y el Profesorado en 
Educación" (p.70). No es sino hasta 1985 cuando se funda la facultad de educación de dicha universidad.

En el caso de Honduras, después de décadas de formación de maestros a través de las escuelas normales, y de alguna manera previendo lo que para Salgado (2004) era "una proliferación incontrolada de instituciones educativas con este mismo fin en todo el país" (párr. 2), se toma la decisión, para el año 1956, de crear la Escuela Superior del Profesorado Francisco Morazán (ESPFM), lo que inicia la formación de docentes especialistas con las características necesarias para laborar exclusivamente en el nivel medio, dejando sin opciones reales de formación de docentes universitarios.

Debido al antecedente que limita la formación de docentes universitarios, para 1964, se da la instauración de la carrera de pedagogía en la Universidad Nacional Autónoma de Honduras (UNAH), esto, debido a los requerimientos del sistema educativo de la época que exigía mayores niveles de conocimiento y metodologías de enseñanza.

Con la creación del Consejo de Educación Superior y su consecuente ley de Educación Superior, en el año 1982, se decreta que las únicas instituciones del nivel autorizadas para la formación de docentes (primaria, secundaria y educación superior) serán la Universidad Nacional Autónoma de Honduras (UNAH) y la Universidad Pedagógica Nacional Francisco Morazán (UPNFM).

A partir de 1989 -que se da la reforma a la actual ley de Educación Superior- y hasta la fecha, la responsabilidad de la formación de docentes en el país ha recaído sobre la UNAH y la UPNFM limitando las opciones en cuanto a cobertura, alcance y diversidad de carreras relacionadas con la formación de docentes, pues según Del 
Cid (2010) "existen docentes [sin el adecuado proceso de formación inicial en Honduras] que no cuentan con la formación científica y pedagógica adecuada para ejercer su función como gestor de la educación". (p.9)

Todo lo anterior genera una serie de preocupaciones a nivel de gobierno, sociedad civil, magisterio y asociaciones gremiales en cuanto al nivel de cobertura, alcance y diversidad de las carreras de formación docente por ofrecer, tomando en cuenta que aún en el país la cobertura de la educación superior no alcanza niveles óptimos y según el Informe Estado de la educación en Honduras, según Salgado y Rápalo. (2012) "la cobertura de la población en edad para estudiar a nivel de educación superior se ha incrementado de $9.8 \%$ en 1997 a 14.6\% en 2009", (p.192), refiriéndose a personas que se encuentran matriculados en una de las veinte universidades; de las cuales seis son públicas y catorce son privadas; sin embargo, la cobertura sigue siendo baja comparada con otros países de Centroamérica.

\section{Las reformas educativas en Honduras y sus efectos en la formación docente}

Si bien es cierto, la Escuela Superior del Profesorado inició funciones en el año de 1956, y no es sino hasta 33 años después que se inaugura la Universidad Pedagógica Nacional Francisco Morazán; los cambios generados se han visto opacados por la interferencia de la clase política y magisterial. Varios han sido los intentos de generar reformas educativas que permitan al sistema educativo nacional estar a tono con lo que sucede en la región; reformas que permitan realmente crear mejoras en los sistemas de formación de formadores.

Desde que se iniciaron los intentos de reformas educativas en el sistema nacional de educación de Honduras, muchos han sido los obstáculos que se han encontrado en el camino para lograr ejecutar los planes propuestos. La ley que estaba en vigencia hasta 2012, data del año 1960. Como parte de los procesos de análisis, a 
finales de la década de los ochenta, se dan una serie de reformas que inician con el Proyecto Eficiencia de la Educación Primaria que tuvo su momento de implementación entre 1987 hasta el 1992. Posteriormente, mediado por presiones sociales, se da la presentación del Programa de Modernización de la Educación Hondureña que tuvo un tiempo de implementación de 10 años, finalizando en el 2002.

Dicho programa de modernización contenía un apartado exclusivo para la formación y capacitación de los docentes y planteaba dentro de sus objetivos la consolidación y modernización de la carrera docente a través de la aprobación del Estatuto del Docente; la profesionalización de los docentes de educación media; y la capacitación de los docentes en servicio. Uno de los mayores obstáculos vividos en los procesos de modernización han sido relacionados con problemas entre magisterio y gobiernos, tal y como apunta Flacso (2008):

Estas iniciativas pretenden reformar lo que ya está reformado y de repente mejorarlo. Sin embargo, esos procesos son verticales omniscientes, sin la participación de ningún sector en el país, por tal motivo, esas reformas han estado condenadas al fracaso, porque no han contado con la participación de los docentes, no tienen ni siquiera los mínimos aportes del gremio magisterial. (p.70)

Ya para inicios del año 2000 , se pone en marcha lo que sería la propuesta de transformación de la educación nacional, impulsada por el FONAC (Foro Nacional de Convergencia Nacional); sin embargo, todos estos intentos de modernización y mejora del sistema educativo nacional se han visto empañados por los constantes roces y conflictos entre la dirigencia magisterial, los docentes y los diferentes gobiernos, lo que ha generado que dichas reformas no avancen y permitan, con esto, el desarrollo en temas relacionados con la formación docente. 
Parte de la problemática que se da debido a los constantes enfrentamientos entre los docentes y los gobiernos han impedido de una u otra manera que la educación avance tal y como lo demandan los cambios socioeconómicos y culturales que se dan en los diferentes contextos latinoamericanos, dejando en total desventaja al país y a las generaciones que se forman en el sistema educativo nacional, tal y como lo menciona el estudio realizado por la PREAL/FEREMA (2002) que dice que:

En cuanto a la formación docente es preciso indicar que Honduras es uno de los pocos países en que la enseñanza en primaria pueden brindarla, en contextos y situaciones normales, personas con secundaria -en la lógica de las antiguas escuelas normales-, siendo ésta una modalidad del nivel medio. En la actualidad hay en el país 13 escuelas normales. (p.54)

En Honduras, con la aprobación de la Ley Fundamental de Educación se propone, entre otras cosas, la profesionalización de los docentes del país, ya que estipula que hasta el 2017 trabajarán los maestros con título medio, y a partir del 2018 todas las personas que laboren dentro del sistema de educación deberán poseer un título universitario. Al mismo tiempo prevé que las universidades del sistema sin importar si son públicas o privadas podrían formar profesionales docentes.

Se pronostica que para el tema de las escuelas normales, aún existentes en el país se conviertan en centros de educación superior. La nueva ley fundamental de educación genera la visión de un perfil profesional para los docentes, por lo que se han creado alianzas con Instituciones como la Universidad Complutense de Madrid, que tiene, según Diario la Tribuna (27/11/2011) "el objetivo de conformar un mejor pensum académico que permita convertir las 13 Escuelas Normales de Honduras en universidades, para la preparación de maestros hondureños". 


\section{Retos y desafíos de la formación docente en Honduras}

Parte de los retos y desafíos que enfrenta la formación docente en el país, es sin duda alguna el hecho de tener la demanda social de incluir a todos los docentes sin una formación del nivel terciario en las aulas universitarias, para así lograr lo que busca la nueva ley fundamental de la educación; que todo el cuerpo docente del país tenga al menos un título de pregrado. La pregunta es si ¿Se tiene la infraestructura humana y física para formar a tantas personas? Aquí es donde se observa la poca comunicación entre sectores.

Otro de los retos es lograr generar los cambios que se requieren en la ley de Educación Superior en el país, debido a que esta no permite a ninguna otra universidad que no sea la UPNFM y la UNAH formar docentes, excluyendo a las restantes 18 instituciones de Educación Superior que tiene el país. Ya que según la Constitución de la Republica en su artículo 163 establece que la formación de docentes del nivel educativo hondureño es función exclusiva del Estado, con base en lo cual el Sistema Educativo Nacional está dirigido, por dos Instituciones: la Secretaria de Educación Pública y la Universidad Nacional Autónoma de Honduras, y más recientemente por el Sistema de Educación Superior.

La formación a nivel de posgrados debe sufrir una renovación, pues a nivel de terciario solamente existe una maestría denominada Maestría en Educación Superior, la cual es impartida por UNAH que capacita al personal docente para laborar en las aulas universitarias, pero con la característica que es exclusiva para el personal de dicha casa de estudios, dejando con esto sin la oportunidad a las personas que deseen formarse en docencia universitaria. Si bien es cierto, la UPNFM tiene programas de posgrado abiertos al público, estos han sido considerados exclusivos para el nivel medio. 
Existe una enorme carencia de profesionales con formación docente para laborar en el nivel terciario, definitivamente una de las carencias con este tema, es que dentro de las aulas universitarias prevalece la experiencia ante la técnica, pues no se cuenta con el suficiente personal que cuente con este tipo de formación.

Se deben replantear los sistemas relacionados con la formación continua en el país, pues además que no se ha logrado generar la cultura de la participación masiva en estos procesos de formación, los controles y estímulos hacia el docente son mínimos, fortaleciendo con esto la poca participación que se observa. En este sentido la profesionalización de docentes de primaria inicia hasta 1998, generando así una total desventaja del país con referencia a otros países de la región.

Si bien se han generado iniciativas de capacitación a nivel de formación inicial, profesionalización y capacitación, el problema principal radica, según Salgado (s.f), "en que no ha existido una integración estructural de los tres componentes entre sí que hubieran permitido un impacto mayor en el sistema educativo nacional". (p.16).

Al mismo tiempo otro de los factores que afectan, tal y como se menciona en el tercer informe de los Objetivos del Milenio de Honduras, según el Sistema de las Naciones Unidas en Honduras (2010) es "la baja calidad de los procesos de enseñanza y aprendizaje, produciendo deficiencias persistentes en la formación de competencias educacionales en docentes y alumnos y alumnas que resultan esenciales para el desarrollo" (p.30), lo que hace necesario la creación de un sistema de acreditación de la calidad; que entre otras cosas evalué y dé seguimiento al desempeño docente para con esto buscar mecanismos que le permita a los maestros del país mejorar sus procesos de enseñanza-aprendizaje, para el beneficio de la población del país. 
Es fundamental que los procesos de formación de docentes y la implementación de las leyes y reformas en el país dejen de lado los oscuros procesos relacionados a la politización de la educación y la corrupción del sistema educativo. Las plazas de docentes han sido utilizadas para gestar votos de los simpatizantes de los partidos políticos, que muchas veces no son personas con competencias docentes; y en el peor de los casos, esas plazas no se llegan a utilizar nunca, pues son las denominadas fantasmas, tal y como se menciona en el Diario la Tribuna (17/4/12) "durante los últimos años el sistema educativo se ha manejado desde el Congreso Nacional y no desde la Secretaría de Educación (...) son los diputados los que ponen y quitan maestros en los centros de enseñanza".

\section{Conclusiones}

Llama la atención, que a raíz de los procesos de independencia en los diferentes países de América Latina, las demandas sociales fueron en aumento y con esto las necesidades de formar docentes que estuvieran en la capacidad de generar procesos de cambio en las personas que educaban. Se puede observar lo fundamental que pudo ser la fuerza laboral de los pedagogos de la época para generar nuevas formas de pensar que estuvieran acorde a las circunstancias.

Similares fueron los momentos y los contextos en los que se dieron los aires de cambio; sin embargo, en algunos países el avance fue mucho mayor que en otros, como el caso de Uruguay que ya para inicios del siglo XX tenía el $100 \%$ de su población de docentes profesionalizada y el caso de Bolivia que hasta inicios del siglo XX formaliza las escuelas normales.

El avance y desarrollo que se observa en los diferentes países analizados en este contexto, puede estar asociado a los esfuerzos que se dieron a finales del siglo XIX 
por profesionalizar a los docentes de la época. No es de esperar que países como Brasil, Argentina, Colombia, Chile y Uruguay sean hoy por hoy potencias y referentes en temas relacionados con formación docente. Definitivamente, los cambios que lograron desarrollar los pioneros por medio de las escuelas normales, ha repercutido sustancialmente sobre las generaciones posteriores.

En el caso de Centroamérica, se observa la situación de países como Costa Rica y Panamá que tomaron las riendas de los procesos relacionados con la formación docente antes de finalizar el siglo XIX, y continuaron ajustando su sistema educativo acorde con los cambios que el avance de los años requería. En el caso de Nicaragua, el gobierno toma tardíamente las riendas de la educación, y no es sino hasta mediados del Siglo XX que formula leyes para regular la formación docente.

Si bien es cierto, el paulatino cambio de las escuelas normales a las Universidades Pedagógicas fue un proceso diferente para los países analizados en este diagnóstico; los cambios observados fueron reflejando poco a poco las reformas que se iban forjando a través de guerras civiles, regularización de los sistemas educativos mediante la implementación de nuevas leyes, el establecimiento de garantías sociales, la apertura de más centros de formación de docentes en las múltiples departamentos, provincias y regiones de los diferentes países de la región, así como la apertura de más centros de educación primaria y secundaria debido al aumento de la demanda.

Otro de los puntos que creemos se debe mencionar es el papel fundamental que jugaron las órdenes religiosas presentes en la región, que previo a la determinación y fundación de las escuelas normales, llevaron el conocimiento necesario para los ciudadanos de los países previo a los procesos independentistas se educaran, 
siempre manteniendo de cerca los procesos de adoctrinamiento, que al final de cuentas era su principal función.

El transcurso de las diferentes situaciones analizadas, no es más que la respuesta ante los procesos de cambio, y por qué no mencionarlo, de la reflexión social que representa la obtención de mejoras en los procesos de formación de docentes a lo largo y ancho de los países que conforman América Latina. Esto conlleva el gradual aumento en la cantidad de escuelas normales en los países que analiza este diagnóstico situacional.

Por otra parte, el aumento de las presiones sociales para mejorar los procesos de formación de maestros en aras de generar cambios significativos en la forma de enseñanza, la llegada de docentes formadores de diferentes países de Europa para contribuir con los procesos de formación de docentes, el Estado toma las riendas de los métodos relacionados a la educación de los maestros, así como la apertura de la carrera de pedagogía en algunas facultades de humanidades de distintas universidades de la región Latinoamericana.

Es necesario que para la implementación de la Ley fundamental de Educación, en Honduras, se destinen los fondos necesarios para lograrlo, pues se debe realizar mucho trabajo en temas como cobertura e infraestructura de las dos universidades que se encargan de formar docente; así como la conformación de un sistema de acreditación y control de la calidad que evalúe el desempeño docente. 


\section{Referencias}

Avendaño, A. (2004). Procesos de Formación de los Docentes por parte de Universidades e Institutos Pedagógicos en Ecuador. IESALC. Recuperado de http://unesdoc.unesco.org/images/0014/001404/140489s.pdf

Alves, S. (maio/agosto, 2009). Aprender a ser maestro/a en las Escuelas Normales de Brasil y España en los ochocientos. Cadernos de Educação FaE/PPGE/UFPel I Pelotas [33], 15-35. Recuperado de: http://www.ufpel.edu.br/fae/caduc/downloads/n33/01.pdf

Aranda, F. (2006). Presentación por escrito de la revisión bibliográfica. Secretaría de Ciencia y Técnica, Universidad Adventista del Plata. Recuperado de http://www.google.hn/url?sa=t\&rct=j\&q=que\%20es\%20la\%20revision\%20bi bliografica\&source $=$ web\&cd=6\&sqi=2\&ved=0CEMQFjAF\&url=http $\% 3 \mathrm{~A} \% 2 \mathrm{~F}$ \%2Fwww.uap.edu.ar\%2Fes\%2F\%3Faccion\%3Dmostrar archivo\%26id arc hivo\%3D1155\&ei=YzCQT42YM4SQ9gTM5tmcBA\&usg=AFQjCNFxEuCphA 8h-cQ-RtOA6ZgjY00UuQ

Ávalos, B. (2003). La formación inicial en Chile. Recuperado de: http://www.ub.edu/obipd/PDF\%20docs/Aspectes\%20laborals/Documents/La \%20Formacion\%20Docente\%20Inicial\%20en\%20Chile.\%20AVALOS.pdf

Báez, M. (2004). Las escuelas normales de varones del siglo XIX en Colombia. Revista Historia de la Educación Latinoamericana, 6, 179-208. Universidad Pedagógica y Tecnológica de Colombia. Recuperado de http://redalyc.uaemex.mx/src/inicio/ArtPdfRed.jsp?iCve=86900611

Cámpoli, O. Michati, M. y Gorboff, N. (2004). La formación docente en La República Argentina. Instituto Internacional para la Educación Superior en América Latina, IESALC. Recuperado de http://www.oei.es/docentes/info pais/informe formacion docente argentina iesalc.pdf

Caraballo, D. Chichiraldi, C. y Curto, V. (2004). La formación docente en el Uruguay. Evolución, estado de situación y perspectivas. IESALC/UNESCO. Recuperado de http://www.glp.net/c/document library/get file?p | id=10413\&folderld=1285 8\&name=DLFE-1250.pdf 
Del Cid, K. (2010). La formación docente y la calidad de la educación en los centros educativos bilingües del municipio de El Progreso, departamento de Yoro (Tesis de Maestría, Universidad Pedagógica Nacional Francisco Morazán, Honduras). Recuperada de http://www.upnfm.edu.hn/bibliod/images/stories/Tesis/karen arely del cid medina.pdf

Diario la Tribuna. (2011). Diputados piden aplicar ley ante corrupción educativa. Recuperado de http://www.elheraldo.hn/SeccionesPrincipales/Pais/Diputados-piden-aplicar-ley-ante-corrupcion-educativa

Diario la Tribuna. (2011). Universidad Complutense apoyará la conversión de Escuelas Normales en Honduras. Recuperado de http://old.latribuna.hn/2011/09/27/universidad-complutense-apoyara-laconversion-de-escuelas-normales-en-honduras/

Instituto Internacional de la UNESCO para la Educación Superior en América Latina y el Caribe. IESALC. (2006). Informe sobre la Educación Superior en América Latina y el Caribe. 2000-2005. La metamorfosis de la educación superior. Caracas, Venezuela.

FLACSO. (2008). Las reformas educativas en honduras. Capítulo 3. Recuperado de http://flacsoandes.org/dspace/bitstream/10469/1979/7/05.\%20Cap\%C3\%AD tulo\%203.\%20\%20Las\%20reformas\%20educativas\%20en\%20Honduras.pd $\underline{\mathrm{f}}$

Lozada, B. (2004). Procesos de formación de los docentes por parte de Universidades e Institutos Pedagógicos en Bolivia. Instituto Internacional de la UNESCO para la Educación Superior en América Latina y el Caribe Recuperado de http://www.radu.org.ar/Info/15\%20form\%20doc\%20bolivia.pdf

Lucio, R. (2008). La cuestión docente: Nicaragua, desde el pasado y el presente hacia el futuro. 1a ed. - Buenos Aires: Fund. Laboratorio de Políticas Públicas. Recuperado http://www.google.hn/url?sa=t\&rct=j\&q=formacion\%20docente\%20nicaragu a\&source =web\&cd=9\&ved=0CF4QFjAI\&url=http\%3A\%2F\%2Fwww.forolatino.org\%2Fflape\%2Fproducciones\%2Fcoleccion Flape 08\%2F25\%2520 Nicaragua\%2520Docente.pdf\&ei=fBu1T-TKI4i8gTi7cUC\&usg=AFQjCNF8bHanFSGg6WSDifRC1QBuC7YYww 
Pérez, A. (1998). La cultura escolar en la sociedad neoliberal. España: Ediciones Morata.

PREAL/FEREMA. (2002). Reforma de la educación en Honduras, propuesta de un modelo considerando cuatro componentes prioritarios. Recuperado de http://www.google.hn/url?sa=t\&rct=j\&q=\&esrc=s\&frm=1\&source=web\&cd=2 \&ved=0CFAQFjAB\&url=http\%3A\%2F\%2Fwww.ferema.org\%2Fdescarga\%2 Freforma.doc\&ei=v3XOT8bgMljPgAeszaRh\&usg=AFQjCNG57rMTnKPBem R1faD7EvJMdNtNag

PREAL/FLACSO. (2008). Síntesis de los documentos del proyecto "Apoyo al diálogo y negociación para las reformas educativas de segunda generación". Recuperado de http://www.piedrasanta.com/HTML/21/docs/formaciondocente.pdf

Salazar, J. (2003). Historia de la Educación Costarricense. Editorial UNED y UCR. Costa Rica. pp. 193-266.

Salgado, R. (s.f). La formación docente en Honduras: Transición hacia un nuevo sistema de formación. Recuperado de http://proyecto.unlam.edu.ar/espec/htdocs 1//programas/formacion\%20doce nte/resumenes/Informe\%20-\%20UP\%20-\%20Honduras\%20\%20Sintesis.pdf

Salgado, R. y Rápalo, R. (2012). Estado de la Educación en Honduras. Universidad Pedagógica Nacional Francisco Morazán. Duplicaciones Castro. Tegucigalpa, Honduras.

Salgado, R. (2004). La formación docente inicial, profesionalización y capacitación docente en Honduras: transición hacia un nuevo sistema de formación docente, UNESCO/IESALC. Recuperado de http://www.radu.org.ar/Info/17\%20form\%20doc\%20honduras.pdf

Santamaría, E. (mayo/agosto, 1994). Formación del Docente en Panamá. Revista Interuniversitaria del Profesorado, 20, 65-79, Recuperado de: http://www.google.com/url?sa=t\&rct=j\&q=formacion\%20docente\%20panam a\&source=web\&cd=1\&ved=0CF4QFjAA\&url=http\%3A\%2F\%2Fdialnet.unirio ja.es\%2Fservlet\%2Ffichero articulo\%3Fcodigo\%3D117825\&ei=C5TLT9bH JYam8ATE |2IDw\&usg=AFQjCNGkTZRLFojf-qDPcoDhAjkNvX3lcg

Santillán, M. (2003). Situación y Perspectivas de las Universidades e Institutos Pedagógicos y su Rol en la Formación de Maestros en la Región. 
Recuperado de: http://www.anuies.mx/e proyectos/pdf/unescoresumen2.pdf

Sistema de las Naciones Unidas en Honduras. (2010). Objetivos de Desarrollo del Milenio, Honduras 2010. Tercer Informe de País.

Sol, R. (2004). Diagnóstico sobre la Formación de Docentes en Instituciones de Educación Superior en Costa Rica. Recuperado de: http://www.oei.es/docentes/info pais/informe formacion docente costa ric a iesalc.pdf

UNESCO/OREALC. (2002). Formación docente: un aporte a la discusión. La experiencia de algunos países. Santiago de Chile: Editorial Andros. Recuperado de http://unesdoc.unesco.org/images/0013/001310/131038so.pdf

Vargas, C. (2008). Diagnóstico situacional de la labor docente y de la oferta de formación de educadores rurales en Costa Rica. Revista Educare, XII, N ${ }^{\circ}$ Extraordinario, 79-97. Redalyc. Recuperado de http://www.redalyc.org/redalyc/pdf/1941/194114584013.pdf 